\title{
Self-Organized Hybrid Wireless Sensor Network for Finding Randomly Moving Target in Unknown Environment
}

\author{
Mininath Nighot*, Ashok Ghatol, Vilas Thakare \\ SGB Amravati University, Computer Department, 444602, Maharashtra (India)
}

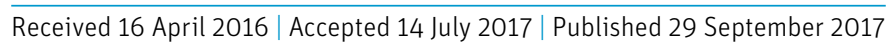

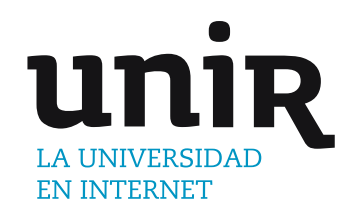

\section{ABSTRACT}

Unknown target search, in an unknown environment, is a complex problem in Wireless Sensor Network (WSN). It does not have a linear solution when target's location and searching space is unknown. For the past few years, many researchers have invented novel techniques for finding a target using either Static Sensor Node (SSN) or Mobile Sensor Node (MSN) in WSN i.e. Hybrid WSN. But there is a lack of research to find a solution using hybrid WSN. In the current research, the problem has been addressed mostly using non-biological techniques. Due to its complexity and having a non-linear solution, Bio-inspired techniques are most suited to solve the problem.

\section{KEYWORDS}

Particle Swarm Optimization, SelfOrganization, Target Finding, Hybrid Wireless Sensor Network.

This paper proposes a solution for searching of randomly moving target in unknown area using only Mobile sensor nodes and combination of both Static and Mobile sensor nodes. In proposed technique coverage area is determined and compared. To perform the work, novel algorithms like MSNs Movement Prediction Algorithm (MMPA), Leader Selection Algorithm (LSA), Leader's Movement Prediction Algorithm (LMPA) and follower algorithm are implemented. Simulation results validate the effectiveness of proposed work. Through the result, it is shown that proposed hybrid WSN approach with less number of sensor nodes (combination of Static and Mobile sensor nodes) finds target faster than only MSN approach.

DOI: $10.9781 /$ ijimai.2017.09.003

\section{INTRODUCTION}

W IRELESS Sensor Networks have gained worldwide attention due to its potential applications in area surveillance such as disaster monitoring, animal monitoring, underwater monitoring etc. [1],[2]. Different sensors have their own physical properties like temperature, moisture, smoke, light, odor, etc. As per the demands of application specific sensors are recommended to be used. The main challenges in WSN are its low bandwidth, memory limitation, and processing power. Researchers need to consider these limitations of WSN to provide a solution. In recent work, very few researchers focused on hybrid WSN due to communication hurdle between SSN to MSN.

Numbers of computations are required in analytical optimization methods. The number of computations depends on the size of the problem. If problem size increases, then computations also increase exponentially. Bio-inspired optimization techniques can be another alternative to analytical optimization. It is more efficient for the increased problem size or when the problem is complex [3],[4],[5].

The objectives of the paper are a) to simulate random moving target searching in an unknown environment, with minimum sensor nodes in hybrid WSN (SSN and MSN), b) to efficiently use, PSO (Particle Swarm Optimization) technique to achieve group movements of MSNs for target searching c) to compare area coverage of all approaches.

* Corresponding author.

E-mail address: imaheshnighot@gmail.com

\section{A. PSO - Particle Swarm Optimization}

Self-organization is one of the important features of Swarm Intelligence (SI). Self-organization is a nonlinear distributed system which cannot have a linear solution and is not controlled by any single particle. It is a continuous process in which particles interact with each other locally [6], [7].

Initially,self-organized systems are predictable, but after some iteration or some time instances, these may be predictable, neutral or unpredictable.

There are mainly five features:

- Positive feedback

- Negative feedback

- Amplification

- Multiple iterations

- Balance of exploitation \& exploration

The system has positive and negative feedback in which positive feedback inspires for the creation of convenient structure while negative feedback neutrals the positive feedback [8],[9],[10].

Multiple iterations are required to reach to the goal. All particles find their own best position (i.e. Local best position). Among the local best positions of all particles, a best position is chosen (i.e. global best position) and all particles use best global position for next movement. 


\section{B. PSO Algorithm}

Generally, PSO performs searching operations using swarm particles. To get the optimal position each move in the direction to their best local position (pbest) and best global position (gbest) [11], [12], [13].

$$
\begin{gathered}
\operatorname{pbest}(i,(k-1))=\underset{k=1,2 \ldots t}{\arg \min }\left[f\left(P_{i}(k)\right)\right], \\
\operatorname{gbest}(k-1)=\underset{i=1,2 \ldots N,}{\arg \min }\left[f\left(P_{i}(k)\right)\right] \\
k=1,2 \ldots \mathrm{t}
\end{gathered}
$$

Where $\mathrm{t}$ is number of iterations and $\mathrm{N}$ is the total number of particles (i.e. swarm size).

Each particle in the swarm decides the movement by an objective function

$$
f(x 1, x 2, \ldots x n) \text { where } f: \mathfrak{R}^{n} \rightarrow \mathfrak{R}
$$

The fitness of particle is calculated from its pbest position in the searching area. The particle where pbest is closer to the gbest have lower cost and vice versa. PSO determines minimization of a fitness function. After each iteration, the position of a particle and its velocity are modified to achieve lower cost or higher fitness value. The notations used in PSO are shown in Table I.

TABLE I. The NOTATIONS USED IN PSO

\begin{tabular}{cc}
\hline Symbol & Meaning \\
$c 1$ & Self-confidence factor \\
$c 2$ & Swarm confidence factor \\
rand 1 \& rand 2 & Random numbers \\
$\omega$ & Inertia weight \\
$X_{i}^{d}$ & Particle's position \\
$V_{i}^{d}$ & Particle's velocity \\
$k, k-1$ & Current and previous iterations \\
pbest ${ }_{i}$ & respectively (movement of particle) \\
gbest & Particles best position \\
$P_{i}$ & Swarm's best position \\
$t$ & Position of agents in the solution space \\
$d$ & Total number of iterations \\
\hline \hline
\end{tabular}

Velocity and position of every particle are modified after $\mathrm{k}$ iteration and is shown as:

$$
\begin{aligned}
& V_{i}^{d}(k)=\omega V_{i}^{d}(k-1)+c 1 \text { rand } 1_{i}^{d}(k) \\
& \quad\left(p b e s t_{i}^{d}-X_{i}^{d}\right)+c 2 \operatorname{rand} 2_{i}^{d}(k) \\
& \quad\left(g b e s t^{d}-X_{i}^{d}\right) \\
& X_{i}^{d}(k)=X_{i}^{d}(k-1)+V_{i}^{d}(k)
\end{aligned}
$$

Here rand $1 \&$ rand 2 are random numbers in the range $[0,1]$ for good coverage. $\omega$ is $0.2 \prec \omega \prec 1.2$ an inertia weight manipulates the trade-off between exploitation \& exploration abilities of the object. Flowchart of PSO is shown in Fig. 1.

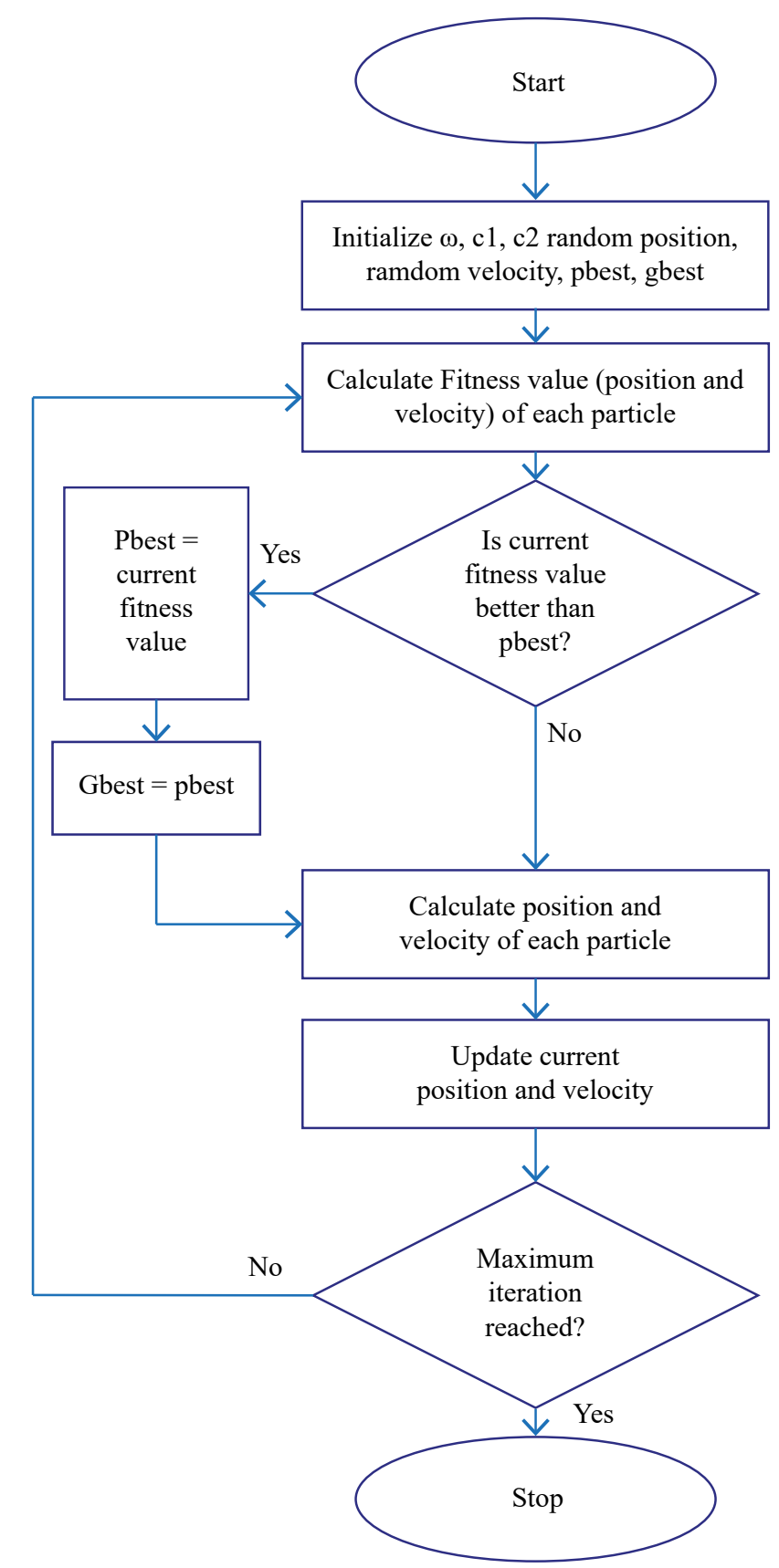

Fig. 1. Working of PSO.

\section{Global Positioning System (GPS) with Real Time Kinematic (RTK)}

GPS is a location receiving device used worldwide. At least four GPS Satellites and a GPS receiver are required to get the location of objects in 2-D space. But its accuracy is 5 meters to 100 meters and precision is 5 meters to more than 20 meters. So use of GPS is not possible in proposed work. It needs more accuracy and precision.

More accurate position can be calculated using Real Time Kinematic (RTK) [14], [15]. Its accuracy is up to 2 centimeters. It consists of one GPS base station and multiple rovers. Setup positions are shown in Fig. 2. GPS base station is positioned on known location. It takes measurements from satellites in view and sends it with its known position to the rovers. Rover receiver also collects measurement from satellite in view, and process it with the base station information. Rover calculates their locations with relative to the base station. 


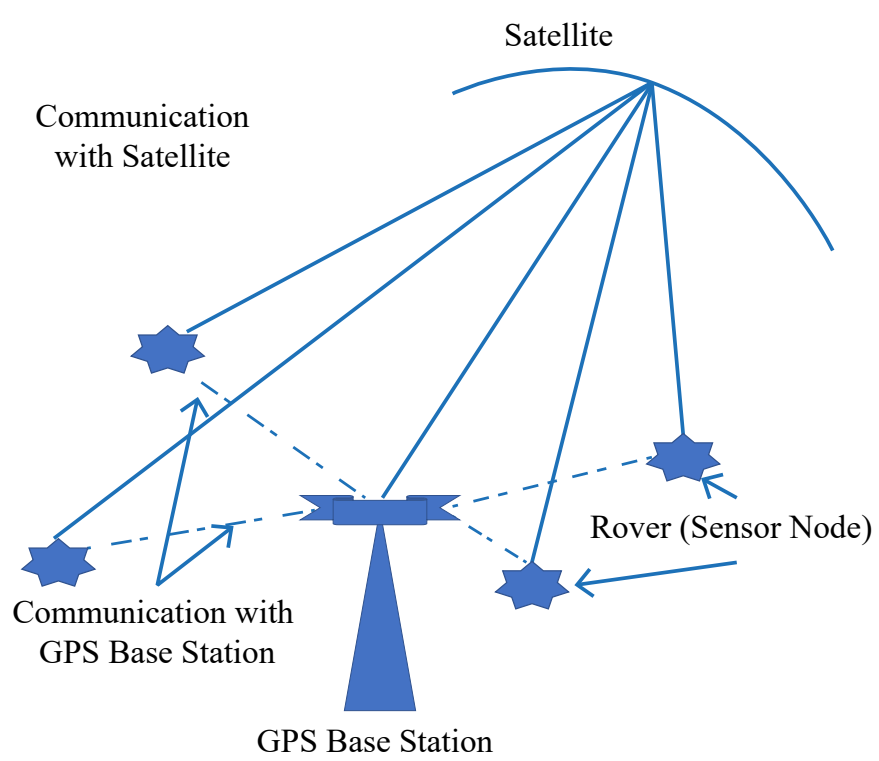

Fig. 2. Real Time Kinematic (RTK) setup.

Equipments of RTK are costlier hence GPS less technique can be used to get the position of sensor nodes. The proposed work is simulation based so not much focused on how to get locations of sensor nodes.

The rest of the paper is organized as follows: A literature survey is explained in section II. In the section III proposed work with algorithms are introduced and explained in detail. Results analysis is given in section IV and in section V conclusion and future scope is given.

\section{Literature SURVEY}

In recent years, several researchers proposed new techniques to find the object. Most of the techniques are SSN based and very few are MSN and hybrid based. Bio-inspired optimization strategies are also implemented to optimize the performance of their work [16],[17]. Some of the techniques are now discussed.

Zhuofan Liao et al [18] proposed algorithms to solve MSN deployment (MSD) problem. Multiple MSN \& Multiple Static Targets are considered in the system. The study focuses on overall energy consumption by minimizing MSN's movement to track the target \& Network connectivity. MSD is divided into two subproblems 1 . TCOV (Target Coverage) and 2. NCON (Network Connectivity) problems. TCOV problem is solved using two Heuristic algorithms- Basic algorithm and TV Greedy algorithm. (Target based Voronoi Greedy algorithm) in which Basic algorithm selects one MSN for one target, and TV-Greedy algorithm minimizes the total number of movements using a Voronoi diagram. For NCON problem ECST \& ECST-H algorithms are proposed to move coverage MSN near to moved MSN. So that moved MSN will be able to communicate with sink node. Matlab simulation is carried out to support the proposed work.

In dynamic transportation system, Ning Zhu. et al [19] proposed a system which is used for collecting traffic information by MSN. Two problems are studied: choosing route link and staying time on the route link. To tackle these problems Ant Colony builds the route for MSN and Particle Swarm Optimization (PSO) determines the stay time on each route link. The proposed work is studied analytically and proved that MSNs are more effective than SSN in transport network surveillance.

Dusade A. et al [20], proposed Moving Object Tracking using Support Vector Machine (MOT-SVM) for finding a movable object by $\mathrm{SSN}$. The proposed algorithm requires fewer communication resources and less amount of communication computations. Because each sensor node needs to send one bit of information to the central processing unit to indicate that a moving object is going far or coming near. Moving target far from observing SSN indicates '-' and near indicates ' + '. Observer SN calculates + and - using RSS (Received Signal Strength). If RSS is less, it is going far \& if RSS is more it is coming near. Experimental analysis is carried out and compared with Aslam's work $\&$ shown MOTSVM performs well in terms of accuracy, precision, and robustness to data errors.

Jia Wei Tang et al [21], proposed a technique to track moving objects using image processing technique. UAV (Unmanned Aerial Vehicle) with vision capability is used to detect the movement of objects. FPGA (Field Programmable Gate Array Algorithm) is used to develop the UAV. For motion estimation and object segmentation block (area based) matching \& RANSAC (Random Sample Consensus) algorithms are used. The entire work is supported by self-experimentation and analytical calculations.

Hamid Maboubi et al [22], [23], focused on problem of tracking and monitoring a mobile target in the field with multiple obstacles for an increasing network lifetime. MSN's are located using relocation technique. The proposed work finds near-optimal relocation strategy for MSN. It also finds the energy efficient path to send information from movable target to destination. The proposed technique is proved by simulation result $\&$ shown network life time increases.

Enyang Xu. et al [24] proposed work for mobile target tracking by MSN. MSN controller gets the location of MSN \& Target continuously by anchor nodes. After analysis of Time of Arrival (TOA), controller guides movement to MSN. MSN navigation strategy, target localization, MSN localization and joint target \& MSN localization are formulated\& calculated. Based on Time of Arrival (TOA), convey optimization algorithm is developed for localization. Cubic law is used for routing MSN.

Yifan Cai et al [25], [26], proposed a couple of algorithms to track the target in a totally unknown physical environment. Multiple robots search the target cooperatively to get the parameter ranges of cooperation method in multiple robots using a combination of HRL and MAXQ algorithms.

All parameters which are required for cooperation are obtained through learning approach and new tasks can be performed by the multiple robots. With simulation study, it is studied and shown that multiple robots in the unknown environment can search the target. Summary of studied literature is shown in Table II. (Table II (a) \& Table II (b)).

\section{Proposed WorK}

Proposed technique shows self-organization Mobile Sensor Node (MSN) and Static Sensor Node (SSN) to find the moving target (T) using Particle Swarm Optimization (PSO). MSN \& SSN are equipped with Global Positioning System (GPS) which is used to tell their exact location coordinates in two-dimensional spaces. Target is assumed to be movable random. Without having visual sense and directional guidance to MSN and SSN, using only location guidance, MSN tracks the moving target.

The proposed system is heterogeneous, using both SSN and MSN in the same system. SSNs have the capability to send their own locations to all MSNs which are far away. If the target is in the searching range (SR) of SSN then SSN sends its own location to all MSN.

MSN are low capability sensors having a movable trolley/vehicle on which MSN's are located. The trolley moves as per the signals are given by MSN. It can move in only three directions Left (Lt), Straight (St) and Right (Rt), from the current position. Left and right rotations are exactly 45 degrees from the current position. Fig. 3 shows the 
TABLE II (a). Summary OF Literature

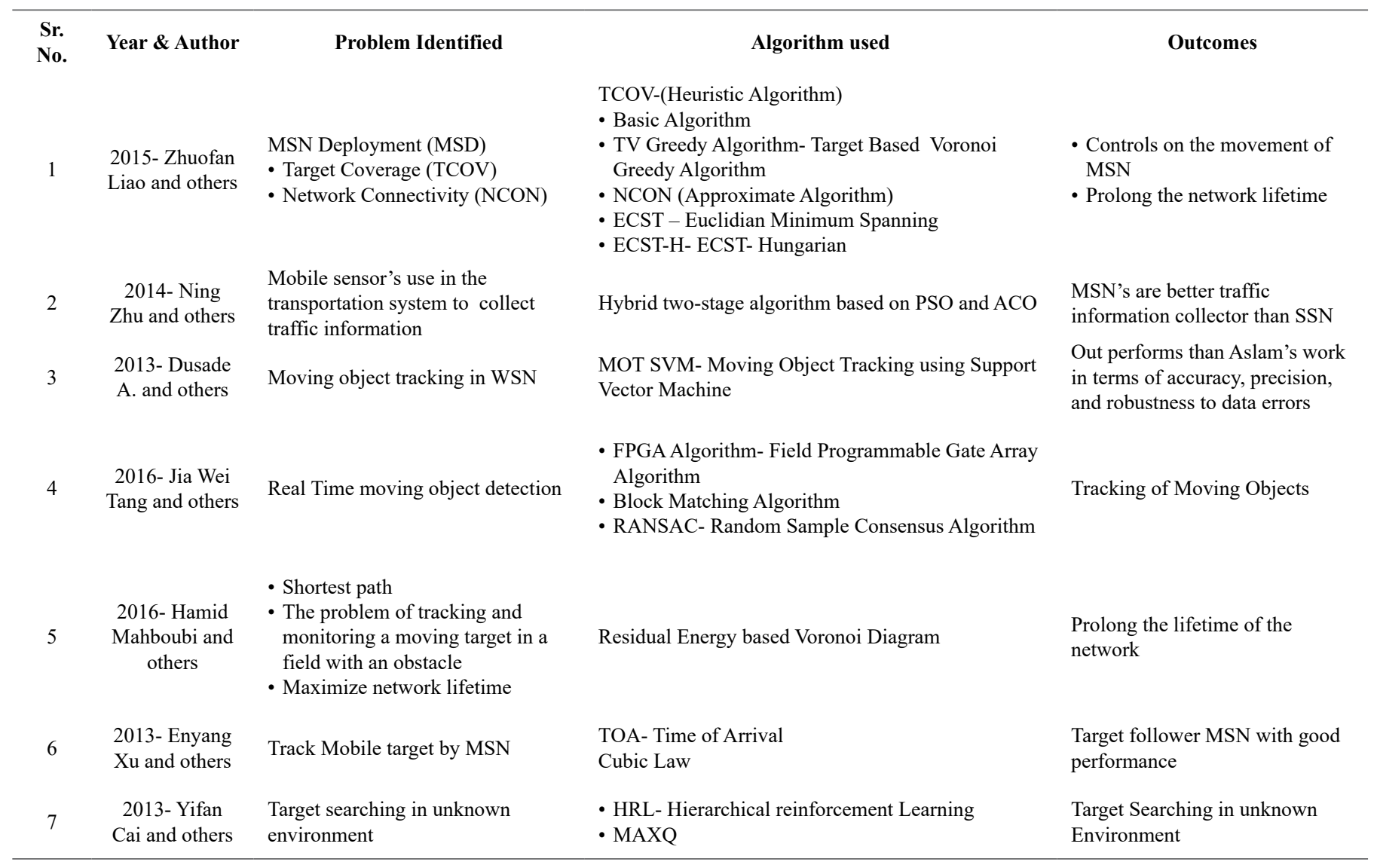

TABLE II (b). Summary of Literature (Continued AS of Table II (a))

\begin{tabular}{|c|c|c|c|c|c|c|c|c|}
\hline $\begin{array}{l}\text { Sr. } \\
\text { No. }\end{array}$ & $\begin{array}{l}\text { Proposed work } \\
\text { compared with }\end{array}$ & $\begin{array}{l}\text { SSN/MSN/ } \\
\text { Hybrid }\end{array}$ & GPS & $\begin{array}{l}\text { Stochastic } \\
\text { Algorithm }\end{array}$ & Target & Network & Control & Simulation/Execution \\
\hline 1 & None & MSN & No & No & Static (Multiple) & MSN & Centralized & Simulation-Matlab \\
\hline 2 & $\begin{array}{l}\text { Self's analytical } \\
\text { results }\end{array}$ & MSN & No & $\begin{array}{l}\mathrm{PSO} \text { and } \\
\mathrm{ACO}\end{array}$ & $\begin{array}{l}\text { No- collect traffic } \\
\text { information }\end{array}$ & $\begin{array}{l}\text { Transport } \\
\text { System }\end{array}$ & Distributed & $\begin{array}{l}\text { Analytical study } \\
\text { (Numerical Experiment) }\end{array}$ \\
\hline 3 & Aslam's Work & SSN & No & No & One - Movable & $\begin{array}{l}\text { Binary Sensor } \\
\text { Network }\end{array}$ & Centralized & Not Mentioned \\
\hline 4 & NO & $\begin{array}{l}\text { UAV/MSN } \\
\text { - Unmanned } \\
\text { Aerial } \\
\text { Network }\end{array}$ & No & No & Movable & $\begin{array}{l}\text { Real Time } \\
\text { Network }\end{array}$ & Centralized & Experimental/Analytic \\
\hline 5 & $\begin{array}{l}\text { No/ Simulation } \\
\text { study compared } \\
\text { analytically }\end{array}$ & MSN & No & No & Movable & $\begin{array}{l}\text { MSN with } \\
\text { obstacle }\end{array}$ & Centralized & Not mentioned \\
\hline 6 & Analytical study & MSN & No & No & Movable - multiple & MSN & Centralized & Not mentioned \\
\hline 7 & NO & $\begin{array}{l}\text { Robots/ } \\
\text { Unknown } \\
\text { Environment }\end{array}$ & No & No & Multiple & $\begin{array}{l}\text { Unknown } \\
\text { Environment }\end{array}$ & Distributed & Not Mentioned \\
\hline
\end{tabular}




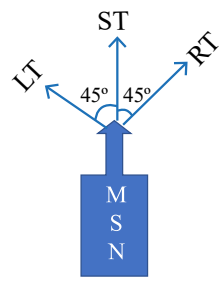

(a)

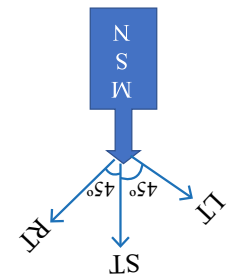

(e)

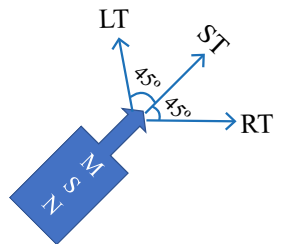

(b)

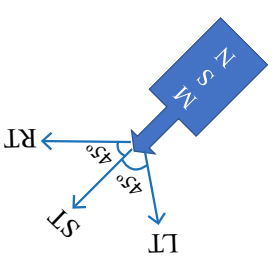

(f)

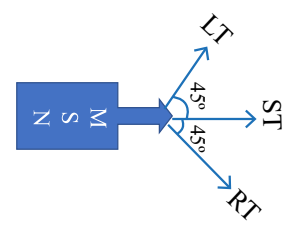

(c)

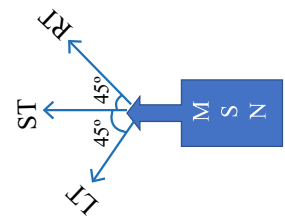

(g)

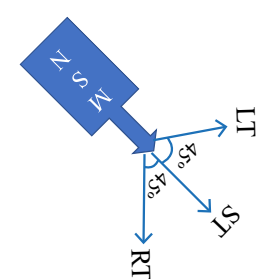

(d)

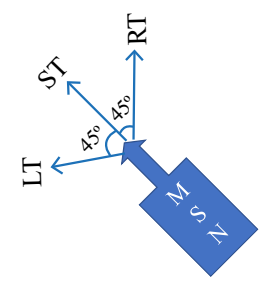

(h)

Fig. 3. The possible rotations from current position.

possible rotations from the current position. There are 8 possible initial positions of MSN and each position has its relative left, straight and right rotations for movement.

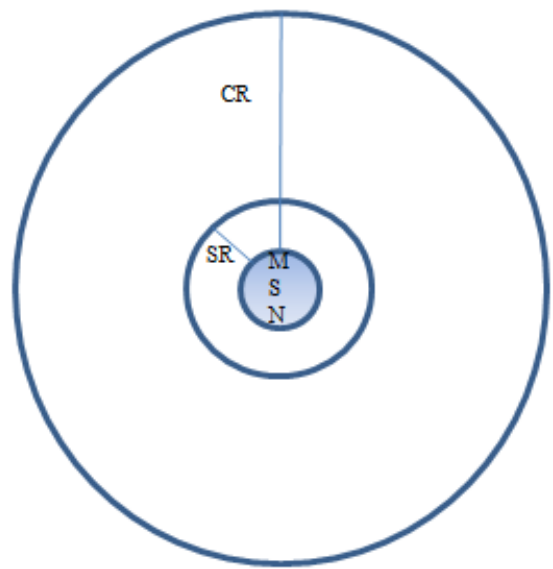

Fig. 4. SR and CR.

Searching Range (SR) is a range or radius of MSN and SSN in which it can search the target. If the target comes in the SR of any $\mathrm{MSN}$, it detects the target. Communication Range (CR) / radio range is a radius of MSN in which MSNs can communicate with each other for sharing their location coordinates and sharing a message mentioning the target is in its SR. [27],[28],[29]. SR and CR are shown in Fig. 4.

\section{A. Topology Formation by MSN}

All MSNs together form a swarm. MSN moves randomly to search the target, but it must be in the range of at least one MSN's CR. Initially MSNs are located in such a way that they will not share each other's SR. Any two MSNs are a 2SR distance away from each other. If two MSNs are sharing SR, then same space will be searched by these two MSNs. By using this initial condition, the search space is increased. Fig. 5 shows the possible minimum and maximum distance between two MSN. Fig. 5 (a) shows MSNs are sharing other's SR. In such case, thesame area will be searched by multiple MSNs and is wastage of time. Fig. 5 (b) shows the minimum distance between two MSN i.e. 2SR. So that it will search in the unsearched area. Fig. 5 (c) shows the maximum distance between two MSN i.e. CR to communicate with each other. If distance is increased than CR then sensors will not be able to communicate with each other.

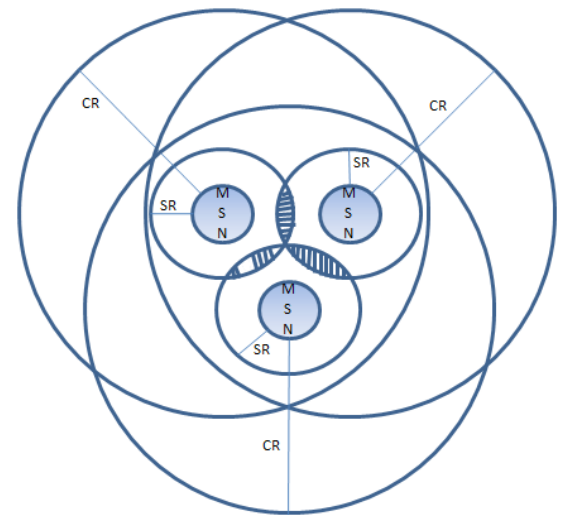

Fig. 5 (a) shows that the dashed area is searched by both sensors which are not required.

\section{B. Basic Flow of Systems}

Initially, SSNs are placed randomly in the searching space. Target (T) is also placed randomly and is movable. It moves randomly and is not GPS equipped. Swarm of MSNs is placed at any random place or at the border of searching space. MSNs move randomly by swarm technique. While moving randomly if $\mathrm{T}$ found, then the mission is complete, else searching process continues.

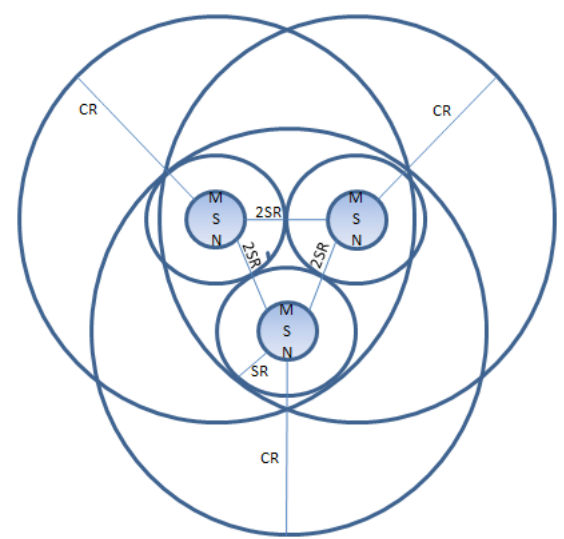

Fig. 5 (b) Shows at least 2SR should be the distance between MSN. 


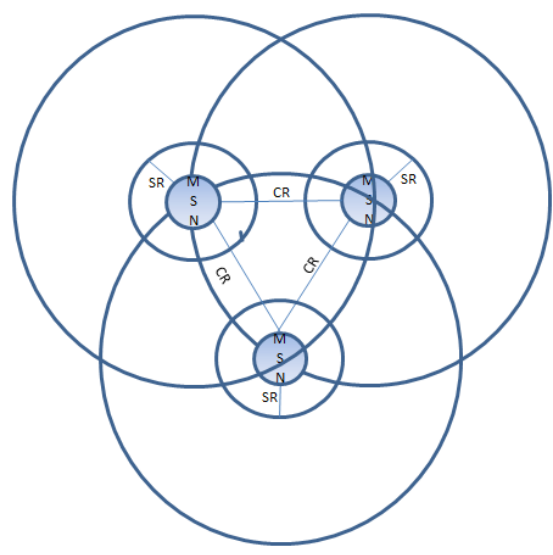

Fig. 5 (c) shows worst case scenario of sensors (maximum distance between two sensors)

Due to the movement of T, if it comes in the SR of any SSN then SSN broadcasts its own location coordinates and a message saying 'Target is found' to all MSNs. Once location and message received by all MSNs, all MSNs calculate its own distance Dist from sender SSN using Euclidean distance [30], [31].

$$
\operatorname{dist}_{i}=d_{i}+n_{i}
$$

Where $d_{i}$ is distance between SSN and MSN and $n_{i}$ is Gaussian additive noise, which has random value uniformlydistributed in the range [32]:

$$
d_{i} \neq d_{i}\left(P_{n} / 100\right)
$$

$P_{n}$ is a percentage noise. Accurate calculation of distance depends on the value of $\mathrm{P}_{n}$

$$
d_{i}=\sqrt{\left(x-x_{i}\right)^{2}+\left(y-y_{i}\right)^{2}}
$$

Where $(x, y)$ are coordinates of SSN and $\left(x_{i}, y_{i}\right)$ are coordinates of particles.

All MSNs share their calculated distance from Sender SSN (SSSN) to each other. The MSN which is closer to SSSN will be selected as a Leader and others will be followers. Leader MSN (LMSN) will decide its direction to reach to SSSN and move step by step towards SSN. Other MSN will not waste their energy for deciding the direction and path. They will just follow the LMSN. At every step, All MSNs calculate their current distance from SSSN and share it to all MSNs. If any Follower MSN (FMSN) is closer to SSSN than LMSN, it will be selected as a new LMSN and former will be FMSN. In such fashion, MSNs travel to SSSN, without knowing directions and any manual interaction. Once all MSNs reach to SSN, they start searching again with their random search.

\section{Proposed Algorithms}

To search the target, four algorithms are proposed which are as follows:

1. MSN's Movement Prediction Algorithm (MMPA) for searching (T) is shown in Fig. 6.

2. Leader Selection Algorithm (LSA) is shown in Fig. 7.

3. Leader's Movement Predication Algorithm (LMPA) for traveling towards SSSN is shown in Fig. 8.

4. Follower's Algorithm (FA) is shown in Fig. 9.

\section{Algorithms}

1. MSN's Movement Prediction Algorithm (MMPA) for searching (T)

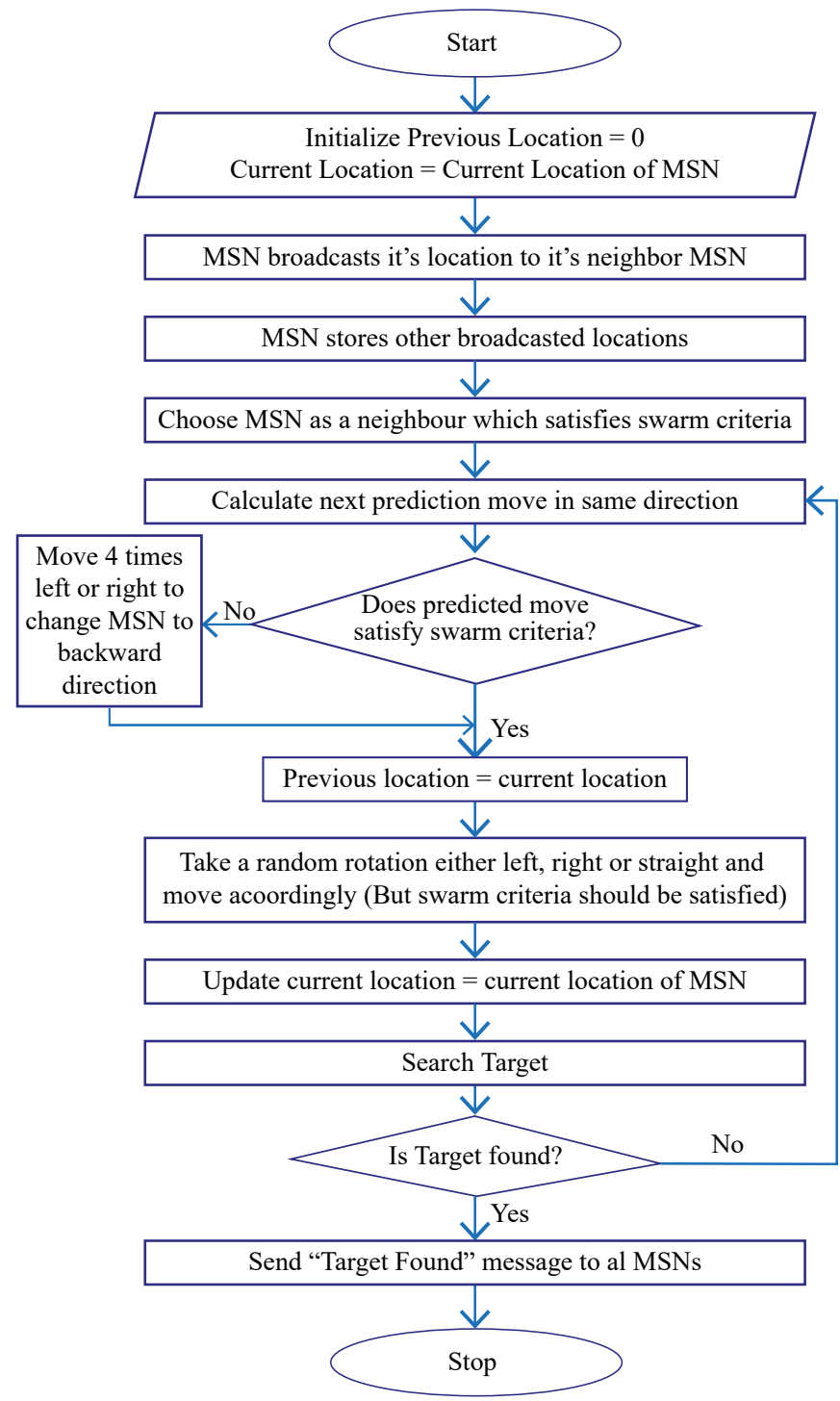

Fig. 6. MMPA Flowchart.

2. Leader Selection Algorithm (LSA)

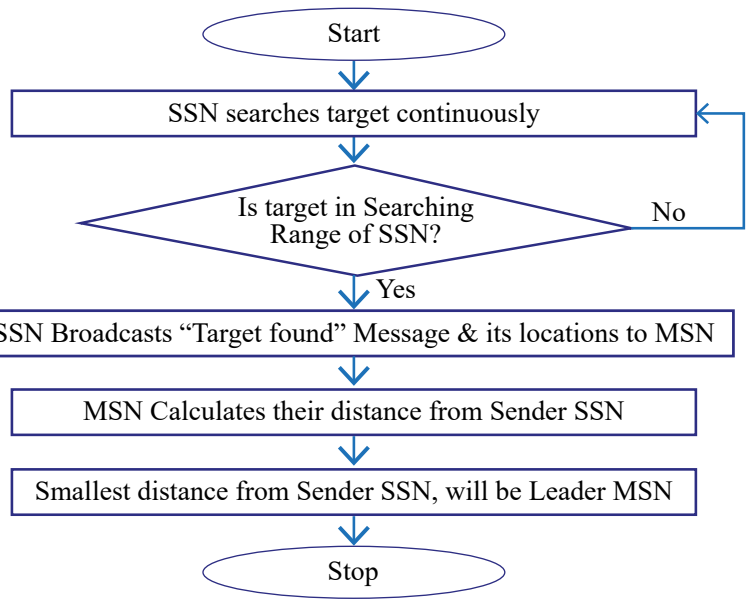

Fig. 7. LSA Flowchart. 
3. Leader's Movement Predication Algorithm (LMPA) for traveling towards SSSN

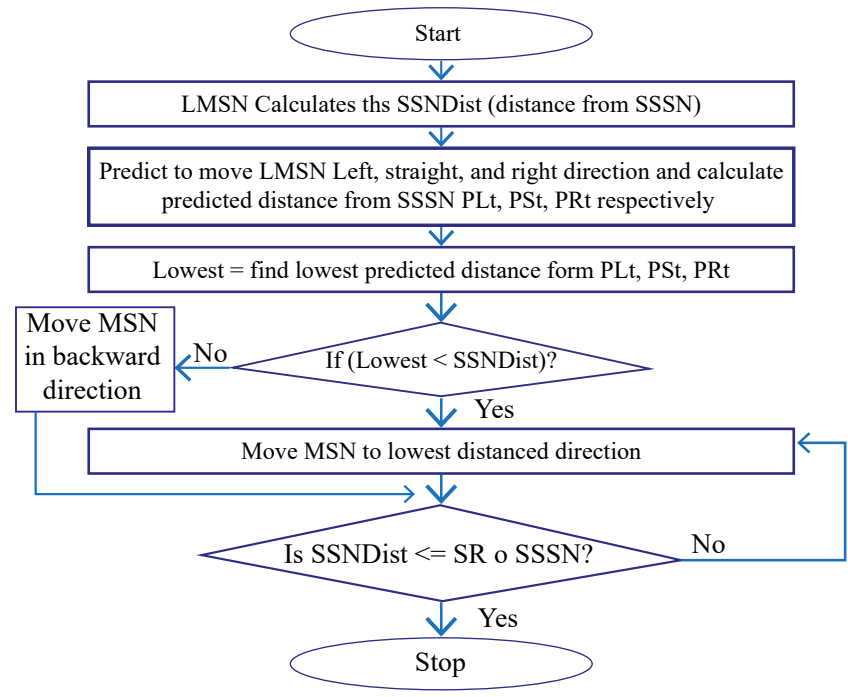

Fig. 8. LMPA Flowchart.

4. Follower's Algorithm

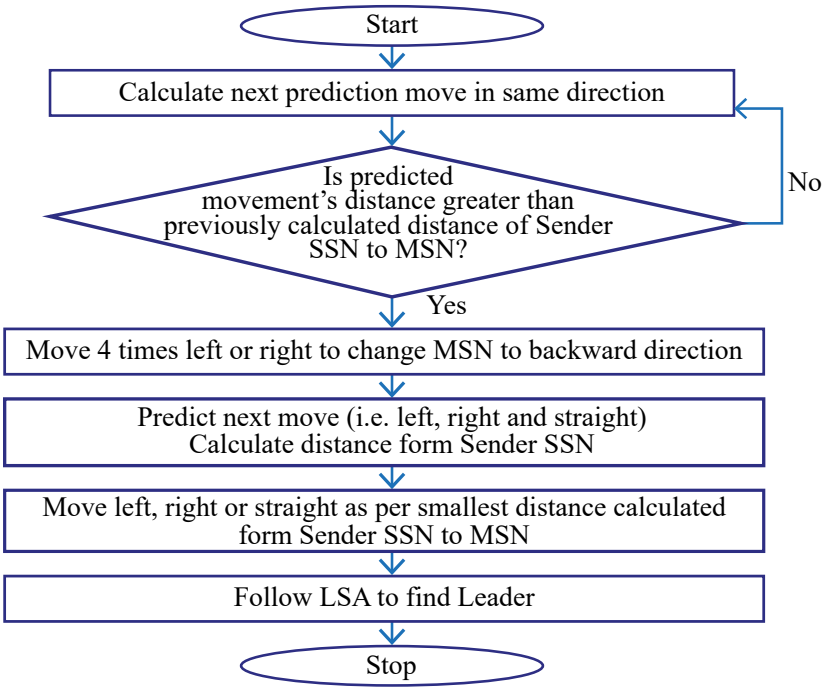

Fig. 9. FA Flowchart.

\section{RESUlts AND Discussions}

Matlab 7.0 simulator is used for the experiment. Due to the random movement of MSNs and T, the number of iterations/steps cannot be predicted. A number of times simulation is executed and tested for finding the $T$. The assumed parameters with respective values for the simulation are given in Table III.

SSNs are placed in searching space in such a way that whole area is covered. Searching space is divided into four quadrants and four SSNs are placed at the center of each quadrant (SSN1- $(2.5,2.5)$, SSN2-(2.5, 7.5), SSN3-(7.5, 2.5) and SSN4-(7.5, 7.5)) and fifth SSN is placed at the center of searching space (SSN5- $(5,5))$. MSNs are placed at leftlower corner of searching the space (MSN1-(0.3, 0.9) and MSN2- $(0.3$, $0.3)$ ). While moving, MSNs maintain at least 2SR distance from each other and will be in the CR of other MSN. T is placed randomly in searching space. The speed of $\mathrm{T}$ is same as speed of MSNs. If the T is in the SR of any MSN then it is assumed that T is found.
TABLE III. Parameters AND Values

\begin{tabular}{lll}
\hline \hline Sr. No. & Parameter & Value \\
1 & Searching Space: & 10 X 10 (Obstacle free) \\
2 & Total Number of MSNs: & 2 \\
3 & Total Number of SSNs: & 5 \\
4 & Total Number of Target (T): & 1 \\
5 & Location of T: & Unknown \\
6 & Communication Range (CR) of sensors: & 1.2 \\
7 & Searching Range (SR) of sensors: & 0.3 \\
8 & Targets Initial Position: & Random \\
9 & MSNs and Target Movement: & Random \\
10 & The speed of Target: & Same as MSN \\
11 & GPS enabled Sensors & Yes \\
12 & Maximum Iterations & 1000 \\
\hline
\end{tabular}

In the simulation, the movement of the target is shown in red colored dots, movement of MSN1 is shown in blue colored dots and movement of MSN2 is shown in green colored dots. Blue circles and pink circles indicate the SR and CR of the respective sensor. In SSN, CR is not visible for the sake of visibility of MSN's and T's movement but it is same as CR of SSN.

Simulations readings are observed after every 100 iterations or $\mathrm{T}$ comes in SR of any SSN or MSN. It is assumed that if T moves in SR of any MSN, then searching mission gets completed and it shows a total number of iterations required to find the $\mathrm{T}$.

A number of times simulation is executed and some of the cases are discussed here as an example.

\section{A. Case 1: Target found by MSNs only}

Fig. 10 (a) shows the initial placement of sensors and T. T moves randomly in searching space as iteration increases. The path and area covered are shown in the red colored tail of T. At the same time MSNs also move using PSO technique to find the T. MSNs maintain the atleast 2SR distance and atmost $1 \mathrm{CR}$ distance from each other. MSNs movements are shown in blue and green colored tail of MSN1 and MSN2 respectively. While moving, MSN found T in their SR at $68^{\text {th }}$ iteration and is shown in Fig. 10 (b).

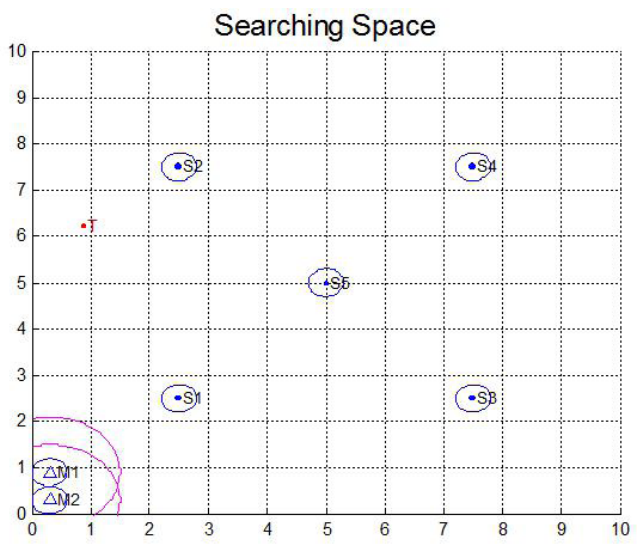

(a) Initialization 


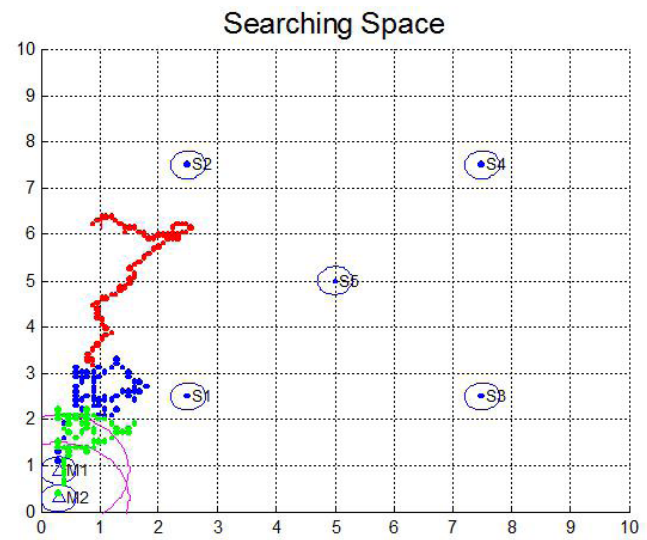

(b) Target is found in 68 Iterations

Fig. 10. Movement of MSNs and T.

\section{B. Case 2: T is Found by MSN with the Help of SSN 4}

Fig. 11 (a) shows the initial localization of sensors and $\mathrm{T}$ where $\mathrm{T}$ is located near the SSN4. After one iteration, T moves in the SR of SSN4, which is shown in Fig. 11 (b). Once T is found by SSN4, it broadcasts 'T Found' message and SSN4's location to MSNs. MSNs stops the searching and switch into the leader-followermode and reaches to $\mathrm{CR}$ of SSN4. It is shown in Fig. 11 (c). While MSNs move towards SSN4, $\mathrm{T}$ moves randomly in another location. Once MSNs reaches in the CR of SSN4, MSNs switch their mode in searching mode and search the T as a random search strategy.

Fig. 11 (d) and 11 (e) show the movements of the sensor and T after $100^{\text {th }}$ and $200^{\text {th }}$ iterations respectively. $\mathrm{T}$ is found in $228^{\text {th }}$ iteration by MSNs and is shown in Fig. 11 (f).

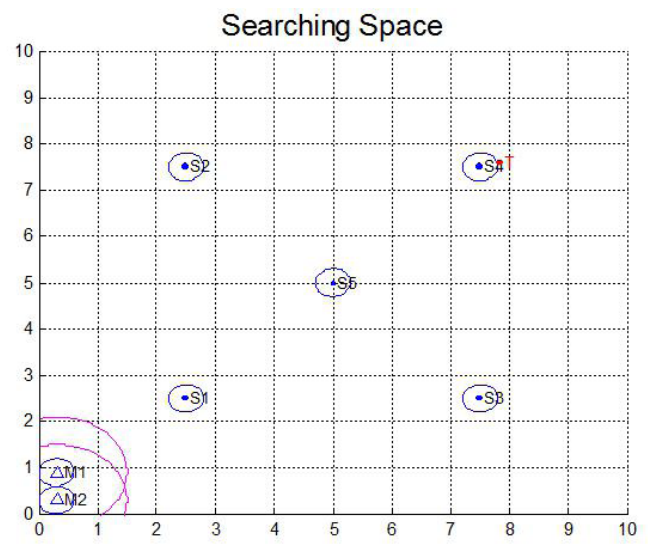

(a) Initialization

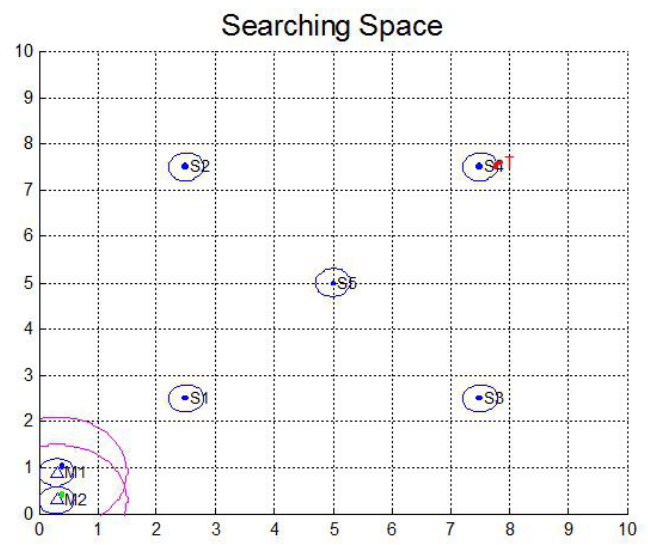

(b) Target is found by SSN 4 in 1 Iteration

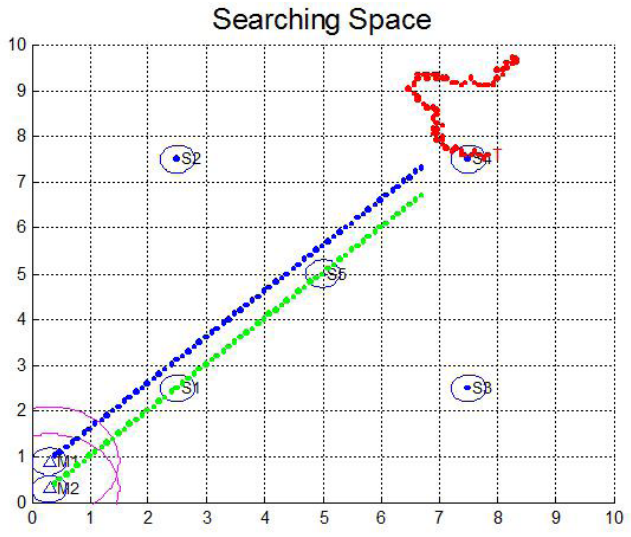

(c) MSNs reach to $\mathrm{SSN} 4$

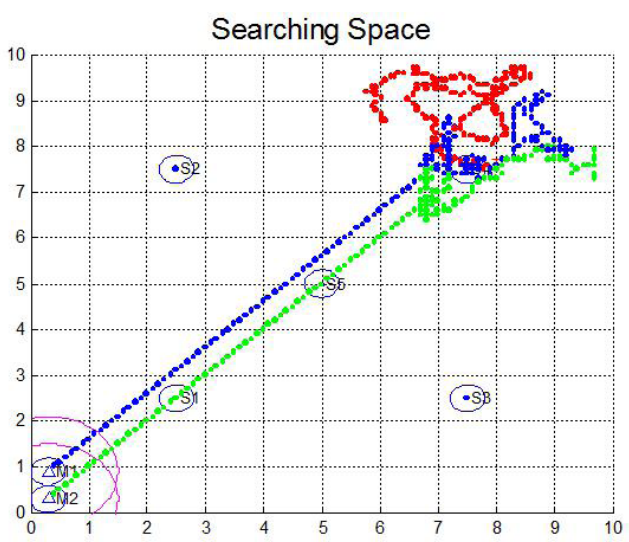

(d) After 100 Iterations

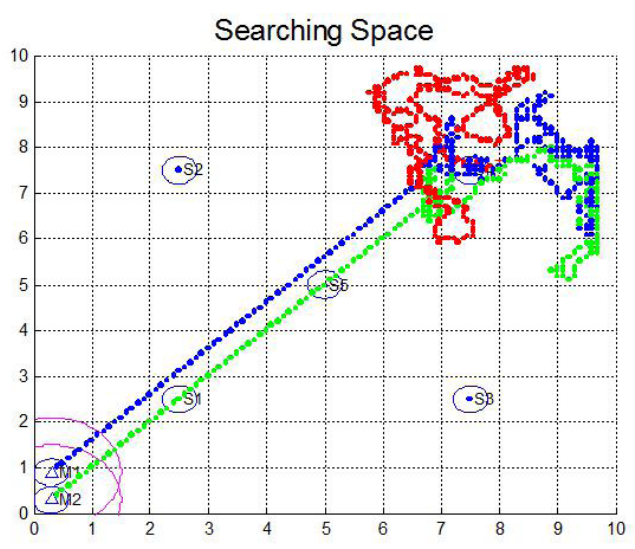

(e) After 200 Iterations

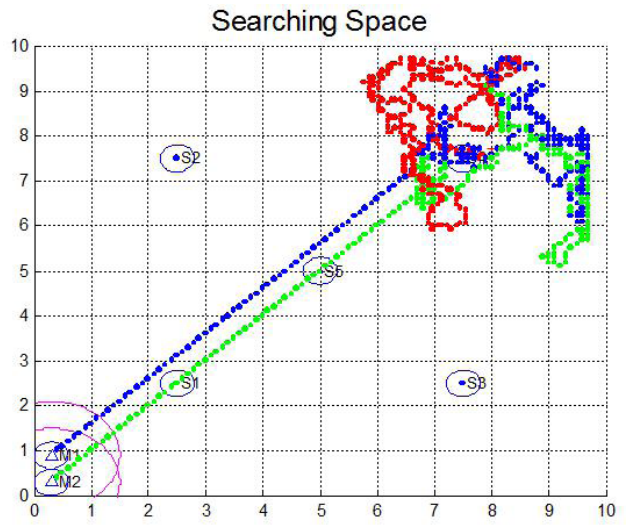

(f) Target found by MSN in 228 Iterations

Fig. 11. Movement of MSNs and T. 


\section{Case 3: T is Found by MSN with the Help of SSN5}

Fig. 12 (a), (b), (c) and (d) are shown as explained in case 2. Instead of SSN4, T appears in SR of SSN5. T is found in the $28^{\text {th }}$ iteration of MSNs.

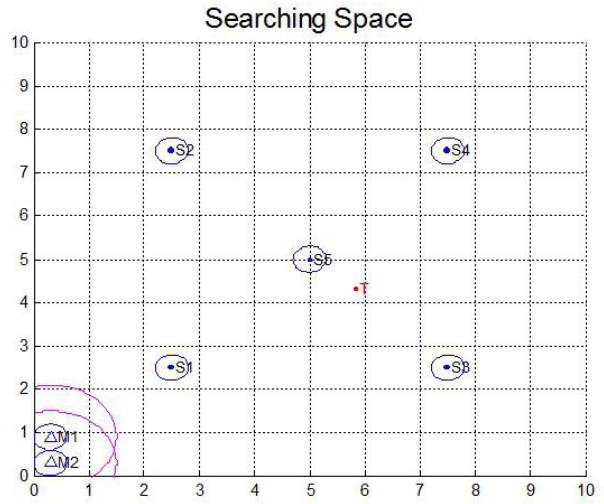

(a) Initialization

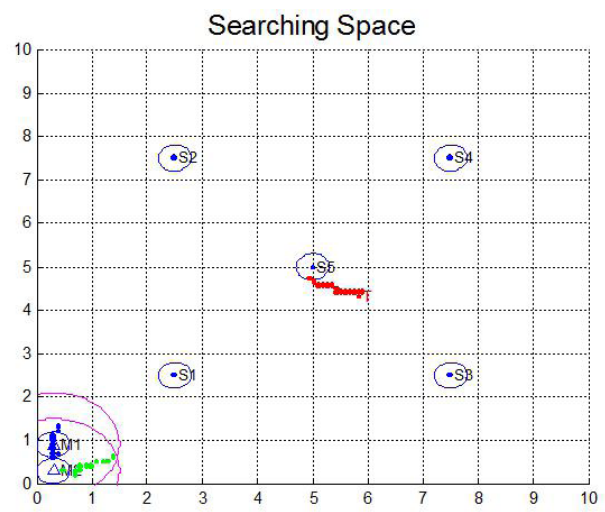

(b) Target Found by SSN5 in 14 Iterations

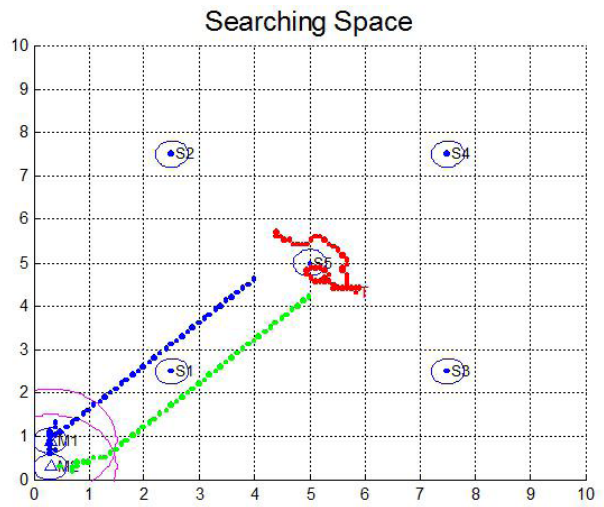

(c) MSN Moves to SSN5

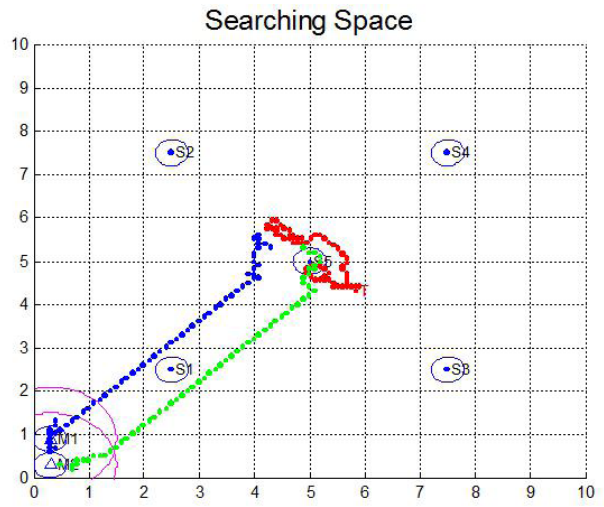

(d) Target is found by MSN in 28 iterations

Fig. 12. Movement of MSNs and T.

\section{Case 4: Involvement of Multiple SSNS}

Sometimes T moves into the bigger area. Before coming all MSNs near to the SSSN, T moves far away from its found location. Once MSNs reaches to SSN, it searches T by random search. So MSNs are

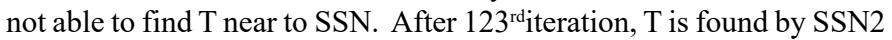
so MSNs need to move towards SSN2.It is shown in Fig. 13 (a), (b), (c) and (d). Fig. 7 (e) shows T is found in $254^{\text {th }}$ iterations by MSN near to SSN2. Even though $\mathrm{T}$ is traveling more distance in a straight way, MSNs are succeeded to find $\mathrm{T}$.

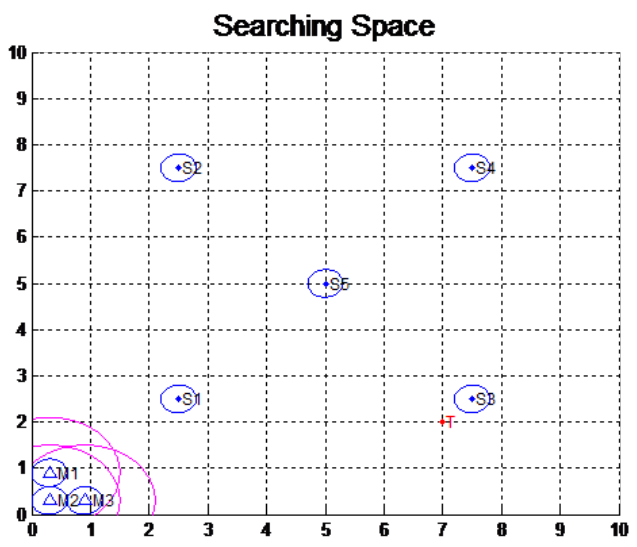

(a) Initialization

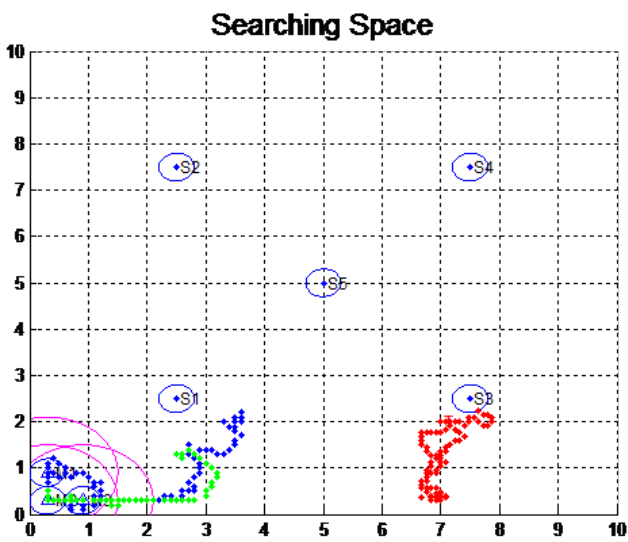

(b) Target is found by SSN 3 in 80 Iteration

Searching Space

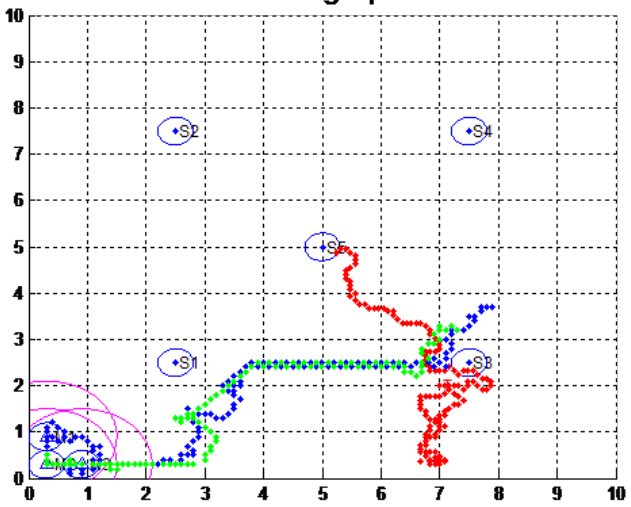

(c) Target is found by SSN5 in 123 Iterations 


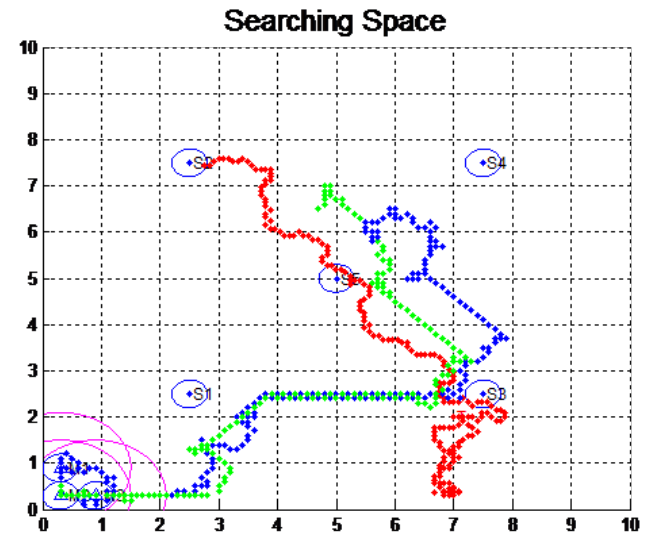

(d) Target is found by SSN2 in 198 Iterations

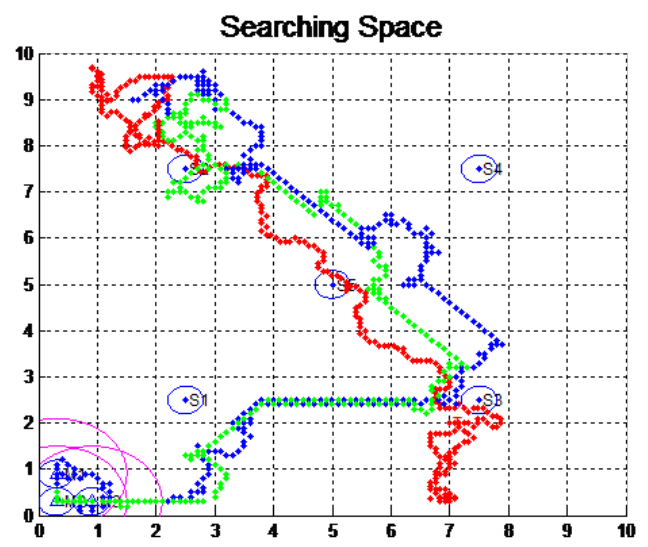

(e) Target found by MSN in 254 Iterations

Fig. 13. Movement of MSNs and T.

\section{E. Case 5: Worst Case}

Sometimes T does not come in SR of any SSN or MSN then it requires more number of iterations. It works like only MSNs are in the network searching for T. Fig. 14 (from the figure (a) to figure (k)) shows that in $1523^{\text {rd }}$ iteration $\mathrm{T}$ is found by MSN.

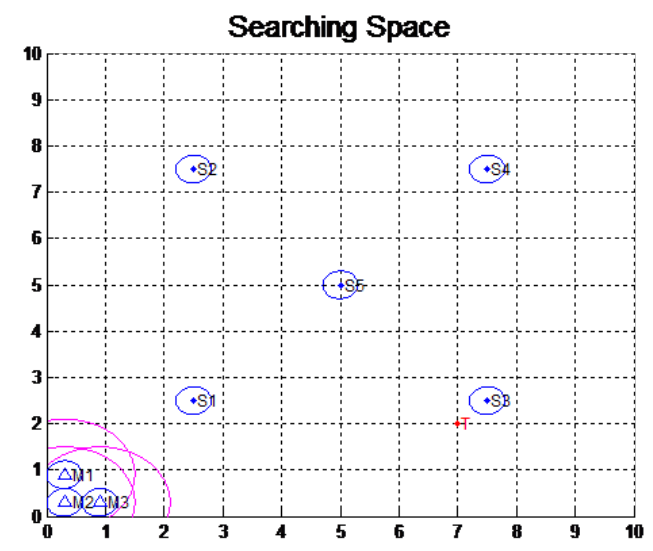

(a) Initialization

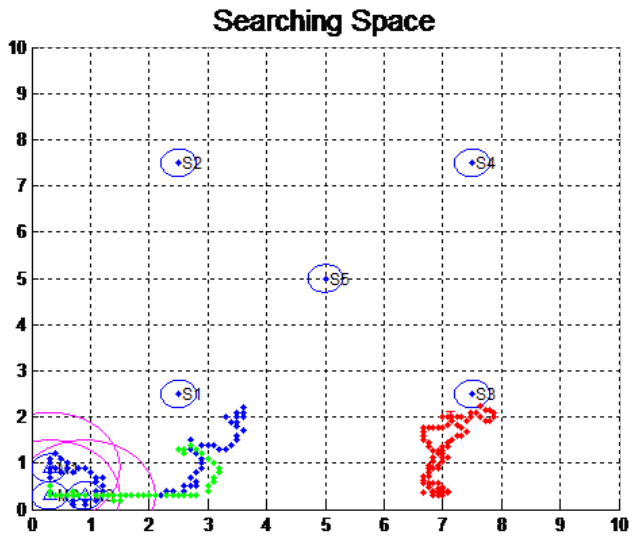

(b) After 100 Iterations

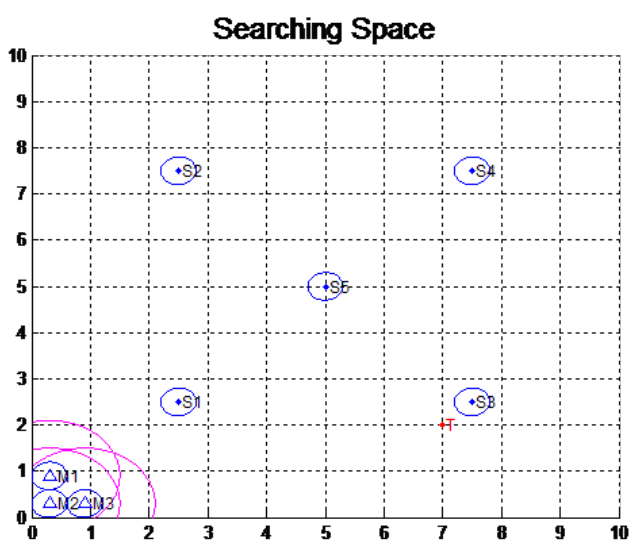

(c) After 200 Iterations

Searching Space

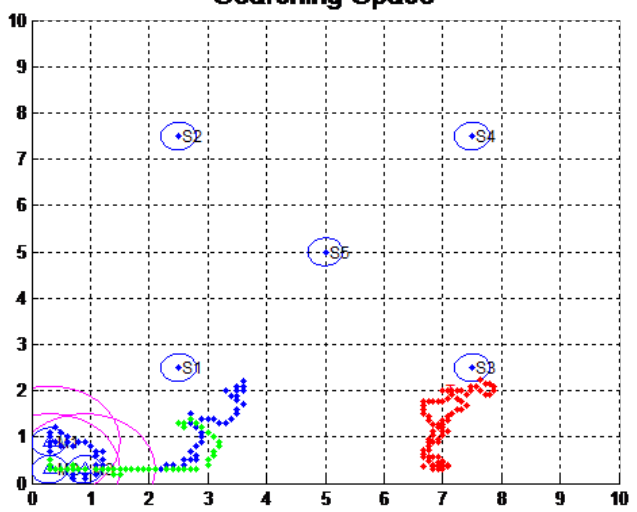

(d) After 300 Iterations

Searching Space

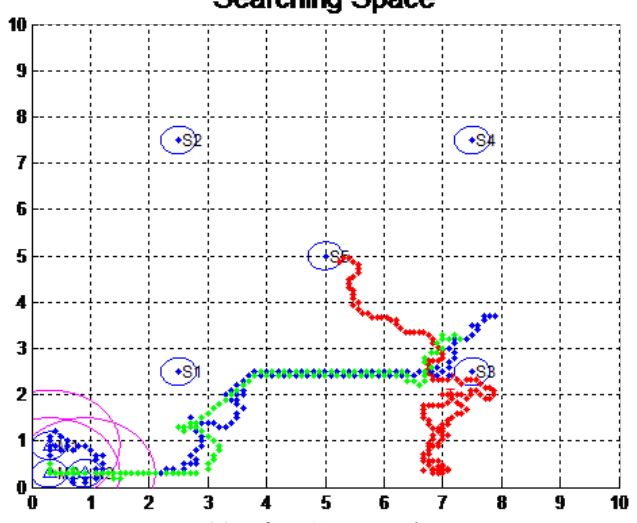

(e) After 400 Iterations 


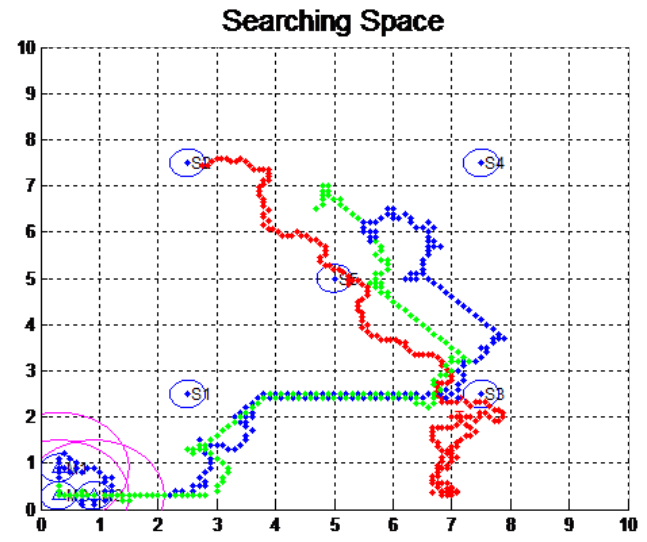

(f) After 500 Iterations

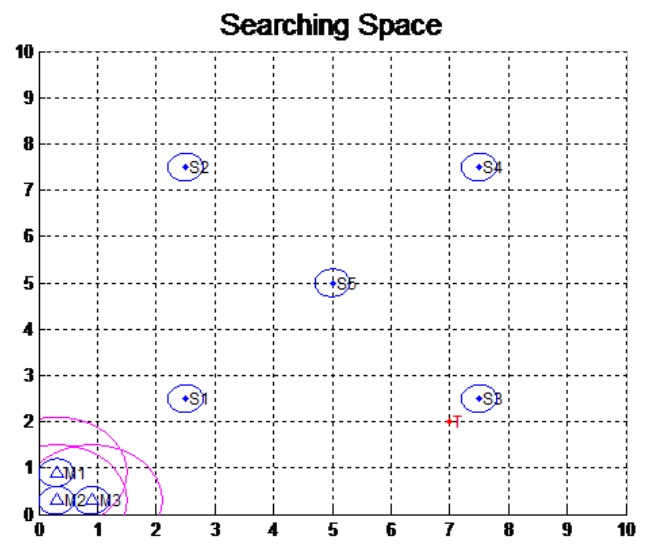

(g) After 600 Iterations

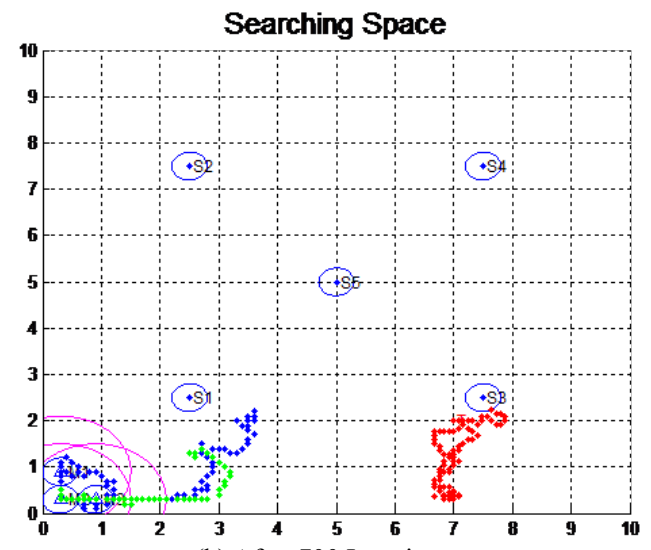

(h) After 700 Iterations

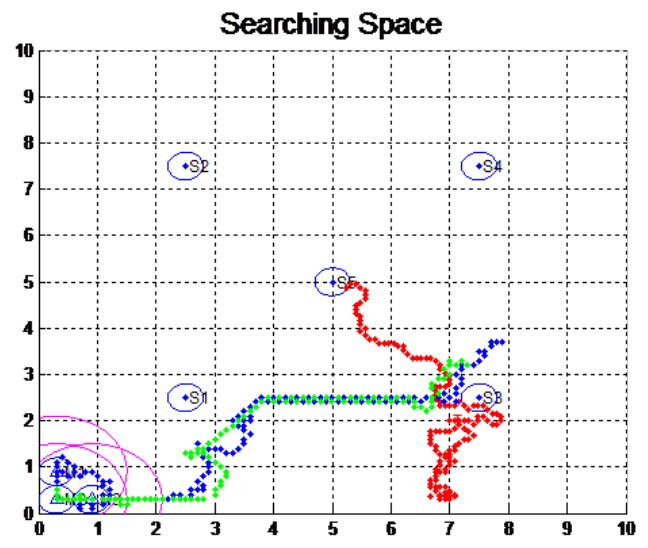

(i) After 800 Iterations

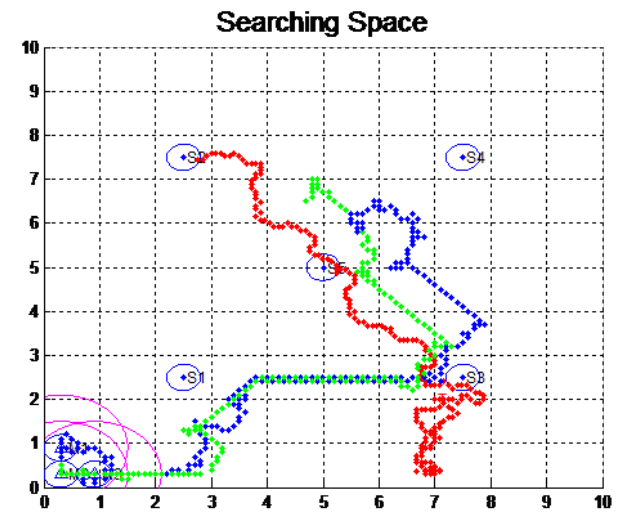

(j) After 900 Iterations

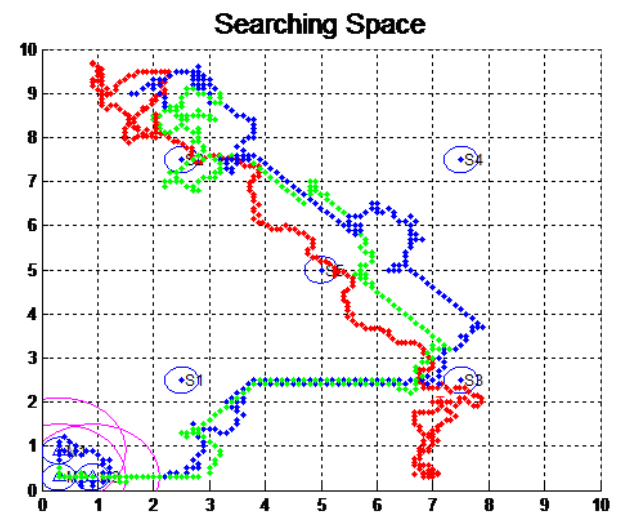

(k) After 1000 Iterations ( $\mathrm{T}$ is found in 1523 iterations)

Fig. 14. Movement of MSNs and T.

F.Comparison Between the Networks with Only MSNs and Hybrid WSN

To show the effectiveness of hybrid WSN, two types of simulations are performed. The first type of simulation consists of only MSNs which are placed on the test bed. And in the second type, both SSNs and MSNs are placed.

If the size of searching space is small then both scenarios work well, but if the size of searching space is large then the simulation with only MSNs requires more number of iterations to find $\mathrm{T}$ as compared to hybrid WSN. The total number of iterations required for both scenarios is shown in Table IV and Fig. 15. To find the T, 10 simulations are carried out of each scenario and numbers of iterations are sorted in ascending order. Average number of iterations in SSN is only 10 but to transmit data to base station it require more energy. Only 572.9 average iterations are required for Hybrid WSN and are far less than only MSN scenario.

TABLE IV. Number of Iterations to FIND T

\begin{tabular}{cccc}
\hline Sr. No. & $\begin{array}{c}\text { Only SSNs } \\
\text { (Dense } \\
\text { Deployment) }\end{array}$ & $\begin{array}{c}\text { Only MSNs } \\
\text { (Number of } \\
\text { Iterations) }\end{array}$ & $\begin{array}{c}\text { Hybrid WSN } \\
\text { (Number of } \\
\text { Iterations) }\end{array}$ \\
1 & 1 & 52 & 28 \\
2 & 1 & 168 & 68 \\
3 & 1 & 1,021 & 228 \\
4 & 1 & 1,021 & 254 \\
5 & 1 & 1,224 & 452 \\
6 & 1 & 1,335 & 622 \\
7 & 1 & 1,512 & 784 \\
8 & 1 & 1,627 & 772 \\
9 & 1 & 1,651 & 998 \\
10 & 1 & 1,724 & 1,523 \\
Average & 10 & 1133.5 & 572.9 \\
\hline
\end{tabular}


Comparison between only MSNs and Hybrid WSN

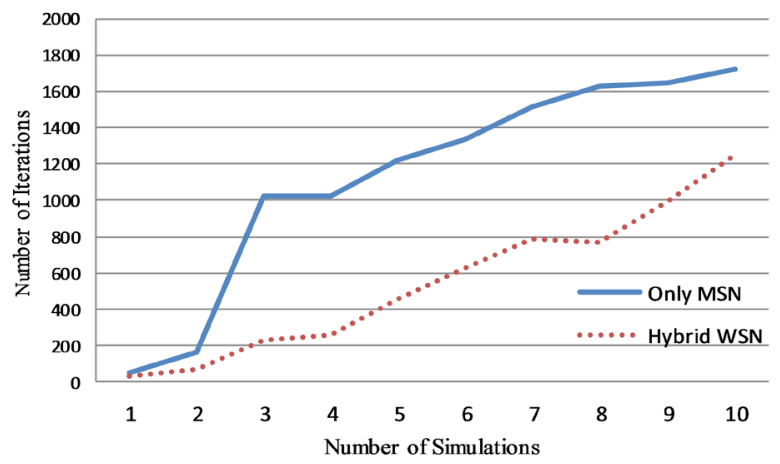

Fig. 15. Effectiveness of Hybrid WSN over the network with only MSNs (SSN is not considered due to dense nodes in network).

In Hybrid approach, if more number of SSNs are placed then the number of iterations reduces.

\section{G. Coverage Comparison of Searching Space}

To cover the complete searching space (10 X 10) by sensors range, more number of SSNs need to deploy. The number of SSNs needed to cover area depends upon the $\mathrm{SR}$ of sensors. If $\mathrm{SR}=0.3$ in same searching space the 289 (17 X 17) SSNs are needed.

\section{1) Degree of coverage}

The degree of coverage can be the ratio of entire searching space and area covered by all sensors and coverage area can be area covered by all sensors trough their SR [18]. In Only SSN approach when $\mathrm{SR}=0.3$, the area coverage, and the coverage degree are 86.7 and 1.15 respectively. In only MSN approach area coverage, and the coverage degree are 0.6 and 166.67 respectively. This approach requires more time to search $\mathrm{T}$.

In the proposed technique SR is 0.3 and number of sensors are only 7 ( 2 MSNs and 5 SSNs). The coverage area and the coverage degree of proposed work are 2.1 and 47.62 respectively, which is shown in Table $\mathrm{V}$ and Fig. 16. Even though proposed work has less coverage area than SSN approach and larger cover area than MSNs approach, it requires less energy than other approaches for searching $\mathrm{T}$.

Table V. Coverage Area and Coverage Degree

\begin{tabular}{|c|c|c|c|c|c|}
\hline Example & $\begin{array}{c}\text { Searching } \\
\text { Space }\end{array}$ & $\begin{array}{c}\text { Number of } \\
\text { Sensors }\end{array}$ & SR & $\begin{array}{c}\text { Coverage } \\
\text { Area }\end{array}$ & $\begin{array}{c}\text { Coverage } \\
\text { Degree }\end{array}$ \\
\hline Only SSNs & $10 \times 10$ & 289 (SSNs) & 0.3 & 86.7 & 1.15 \\
\hline Only MSNs & $10 \times 10$ & 2 (MSNs) & 0.3 & 0.6 & 166.67 \\
\hline $\begin{array}{l}\text { Proposed } \\
\text { work }\end{array}$ & $10 \times 10$ & $\begin{array}{l}7 \text { (2 MSNs } \\
+5 \mathrm{SSNs})\end{array}$ & 0.3 & 2.1 & 47.62 \\
\hline
\end{tabular}

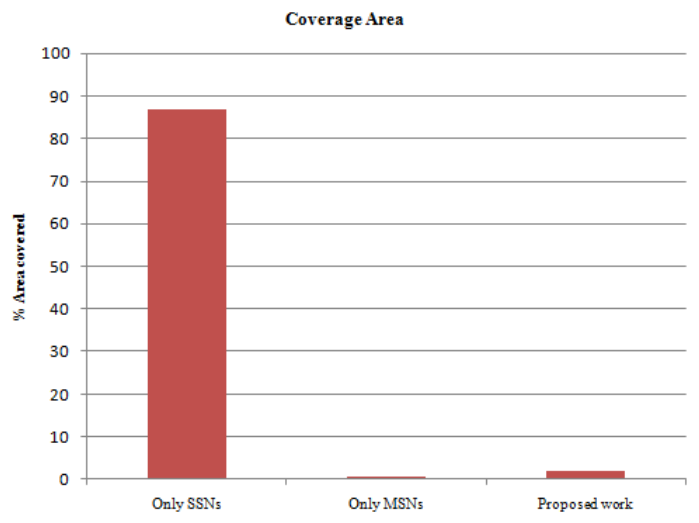

Fig. 16. Coverage Area Comparison.
Inspite of time constraint, even though area covered by proposed approach is 2.1 , it finds target successfully.

\section{Conclusion and Future Scope}

Randomly moving target is efficiently found by novel proposed technique. MSNs are allowed to move in only three random directions. Without control of third parties, MSNs move autonomously and find target successfully. A novel concept of hybrid WSN is implemented to find the target. SSNs and MSNs are utilized together to achieve objectives. If more number of SSNs \& MSNs are deployed, then searching time reduces tremendously. A PSO technique in hybrid WSN is effectively implemented to find the unknown target in an unknown environment. Results show that hybrid WSN performs better than only MSNs in the network.

It is proved that using less number of sensors, target finding can be done effectively so due to less number of sensors utilized in the network, equipment cost and its maintenance cost is reduced.

Obstacle free environment is assumed for simulation. Single target is assumed and its speed is assumed same as MSNs speed. But in real case target's speed may be lower or higher than MSNs speed. In future work, it is planned to consider obstacles in searching space with multiple targets and lower or higher speed of target than MSNs.

\section{REFERENCES}

[1] C.Y. Chong and S. Kumar, "Sensor Networks: Evolution, Opportunities and challenges," proceeding IEEE, vol. 91, no. 8, pp. 1247-1256, Aug. 2003.

[2] Mininath Nighot and Ashok Ghatol, "GPS Based Distributed Communication Protocol for Static Sensor Network (GDCP)," Procedia Computer Science, Vol. 78, pp. 530-536, 2016.

[3] Bahuguna, Y., D. Punetha, and P. Verma, "An analytic Study of the Key Factors Influencing the Design and Routing Techniques of a Wireless Sensor Network," International Journal of Interactive Multimedia and Artificial Intelligence, vol. 4, no. 3, pp-11-15, 2017.

[4] Eliseo Ferrante, Ali Emre Turgut, Alessandro Stranieri, Carlo Pinciroli, Mauro Birattari, Marco Dorigo, "A self-adaptive Communication strategy for flocking in stationary and non-stationary enviroments," Nat. Comput (2014), vol. 13, pp. 225-245, 2014.

[5] Hongliang Ren and M.M.Q.H. Meng, "Biologically Inspired Approaches for Wireless Sensor Networks," Proceeding IEEE International Conference, Mechatronics and Automation, 2006.

[6] Zeng, J.C., Jie, J. and Cui, Z.H., "Particle Swarm Optimization," Science Press, Beijing (2004).

[7] M. K. Nighot, V. H. Patil and G. S. Mani, "Multi-robot Hunting based on Swarm Intelligence," Hybrid Intelligent Systems (HIS), vol. 12, pp. 203206, 2002.

[8] Kennedy, J. and Eberhart, R.C. "Particle swarm optimization," Proceeding IEEE Conference on Neural Networks, vol. 11, pp. 1942-1948, 1995.

[9] J. Kennedy and R. C. Eberhart, "Particle swarm optimization," Proc. IEEE Int. Conf. Neural Network, pp. 1042-1048, Nov.-Dec. 1995.

[10] R.C. Eberhart and J. Kennedy, "A new optimizer using particle swarm theory," Proceedings oninternational symposium on micro machine and human science, IEEE Service Center, USA, pp. 39-43, 1995.

[11] J. Kennedy, "The particle swarm: social adaptation of knowledge," Proceedings of IEEE international conference on evolutionary computation, IEEE Service Center, USA, pp. 303-308, 1997.

[12] C. Anderson and N.R. Franks, "Teams in animal societies behavior," Ecol, 12 (5), pp. 534-540, 2001.

[13] P.B.S. Lissaman and C.A. Shollenberger, "Formation flight of birds Science," 168 (3934), pp. 1003-1005, 1970.

[14] D. Manolakis, "Efficient Solution and Performance Analysis of 3D Position Estimation by Trilateration," IEEE Trans. On Aerospace and Electonic Systems, vol. 32, no. 4, pp. 1239-1248, 1996.

[15] Xiyang He, Xiaohong Zhang, Long Tang and Wanke Liu, "Instantaneous Real-Time Kinematic Decimeter-Level Positioning with BeiDou TripleFrequency Signals over Medium Baselines," Sensors, 16, 1 pp. 1-19, 2016. 
[16] Kaur, R., and S. Arora, "Nature Inspired Range Based Wireless Sensor Node Localization Algorithms," International Journal of Interactive Multimedia and Artificial Intelligence, vol. 4, no. 3, pp-1-11, 2017.

[17] Sanjeev Wagh and Ramjee Prasad "Energy Optimization in Wireless Sensor Network through Natural Science Computing: A Survey," Journal of Green Engineering, River Publication, vol. 3, pp. 383-402, 2013.

[18] Zhuofan Liao, Jianxin Wang, Shigeng Zhang, Jiannong Cao and Geyong Min, "Minimizing Movement For Target Coverage and Network Connectivity in Mobile Sensor Networks," IEEE Transactions on Parallal Distributed System, vol. 26, no. 7, July 2015.

[19] Ning Zhu, Yang Liu, Shoufeng Ma, and Zhengbing He, "Mobile Traffic Sensor Routing in Dynamic Transportation Systems," IEEE Transactions on Intelligent Transportation System, vol. 15, no. 5, October 2014.

[20] Dusadee Apicharttrisorn, Kittipat Apicharttrisorn and Teerasit Kasetkasem, "A moving object tracking algorithm using support vector machines in binary sensor networks," 13th International Symposium on Communications and Information Technologies (ISCIT), pp. 529-534, September 2013

[21] Jia Wei Tang, Nasir Shaikh-Husin, Usman Ullah Sheikh, and M. N. Marsono, "FPGA-Based Real-Time Moving Target Detection System for Unmanned Aerial Vehicle Application," International Journal of Reconfigurable Computing, Vol. 2016, Article ID 8457908, pp. 1-16, 2016.

[22] Hamid Mahboubi, Walid Masoudimansour, Amir G. Aghdam and Kamran Sayrafian-Pour, "Maximum Lifetime Strategy for Target Monitoring With Controlled Node Mobility in Sensor Networks with Obstacles," IEEE Transactions on Automatic Control, vol. 61, no. 11, pages 3493-3508, November 2016

[23] H. Mahboubi, W. Masoudimansour, A. G. Aghdam, and K. SayrafianPour, "Cost-efficient routing with controlled node mobility in sensor networks," in Proceedings of the IEEE Multi-Conference on Systems and Control, pp. 1238-1243, 2011.

[24] Enyang Xu, Zhi Ding, Fellow,IEEE, and Soura Dasgupta, "Target Tracking and Mobile Sensor Navigation in Wireless Sensor Networks," IEEE Transactions on Mobile Computing, vol. 12, no. 1, pp. 177-186, January 2013.

[25] Yifan Cai,Simon X. Yang andXin Xu, "A combined hierarchical reinforcement learning based approach for multi-robot cooperative target searching in complex unknown environments," 2013 IEEE Symposium on Adaptive Dynamic Programming and Reinforcement Learning (ADPRL), pp. $52-59,2013$.

[26] Yifan Cai, Simon X. Yang, Xin Xu and Gauri S. Mittal, "A Hierarchical Reinforcement Learning Based Approach for Multi-robot Cooperation in Unknown Environments," Proceedings of the 2011 2nd International Congress on Computer Applications and Computational Science, vol. 144 of the series Advances in Intelligent and Soft Computing, pp 69-74, 2011.

[27] Yuanjiang Huang, José-Fernán Martínez, Juana Sendra, and Lourdes López, "The Influence of Communication Range on Connectivity for Resilient Wireless Sensor Networks Using a Probabilistic Approach," Hindawi Publishing Corporation, International Journal of Distributed Sensor Networks, vol. 2013, Article ID 482727, pp. 1-11, 2013

[28] Vijay Bhaskar Semwal, Vinay Bhaskar Semwal, Meenakshi Sati, and Dr.Shirshu Verma, "Accurate location estimation of moving object In Wireless Sensor network," International Journal of Interactive Multimedia and Artificial Intelligence, vol. 1 no. 4, pp-71-75, 2011.

[29] F. Xue and P. R. Kumar, "The number of neighbors needed for connectivity of wireless networks," Wireless Networks, vol. 10, no. 2, pp. 169-181, 2004.

[30] L.E. Miller, "Distribution of Link Distances in a Wireless Network," J. Research of the National Institute of Standards and Technology, vol. 106, pp. 401-412, 2001.

[31] S. De, A. Caruso, T. Chaira, and S. Chessa, "Bounds on Hop Distance in Greedy Routing Approach in Wireless Ad Hoc Networks," International Journal Wireless and Mobile Computing, vol. 1, pp. 131- 140, 2006.

[32] Maneesha V. Ramesh, Divya P. L. et al., "Perfomance Enhancement in Distributed Sensor Localization using Swarm Intelligence", 2012 International Conference on Advances in Mobile Network, Communication and its Application, 2012.

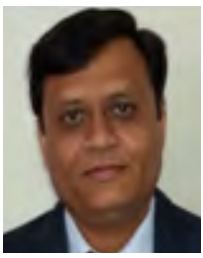

\section{Mininath K. Nighot}

Mininath K. Nighot is perusing Ph.D. in computer science and engineering at Shree Sant Gadgebaba Amravati University, Amravati. He received is master degree in computer engineering from Dr. D.Y. Patil college of engineering, university of Pune, Pune in 2008. He has completed his graduation in computer science and engineering from Babasaheb Naik college of engineering, Pusad, Amravati University in 2002. His research interest includes wireless communication, protocol design for wireless sensor network and effective use of optimization techniques.

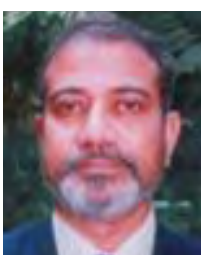

\section{Ashok Ghato}

Ashok Ghatol is a former vice chancellor at Dr. Babasaheb Ambedkar Technological University, Lonere. He was executive committee member and vice president of Indian Society for Technical Education New Delhi. He was chairman of AIB of technical education, AICTE, New Delhi in 2009. He was awarded as best teacher by Maharashtra Government in 1998-99. He earned his Ph. D. degree in electrical engineering from IIT Powai, Mumbai. He wrote three books on different technology. He had guided more than 20 Ph.D. and 30 Master students in engineering. Optimization techniques.

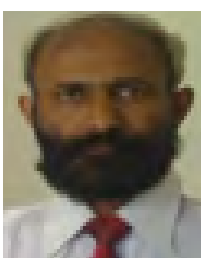

Vilas M. Thakare

Vilas M. Thakare is Professor and Head in Post Graduate department of Computer Science and engg, Faculty of Engineering \& Technology, SGB Amravati university, Amravati. He is also working as a coordinator on UGC sponsored scheme of e-learning and m-learning specially designed for teaching and research. He is Ph.D. in Computer Science/Engg and completed M.E. in year 1989 and graduated in 1984-85. 